\title{
High Speed Rail Transport Valuation
}

\author{
Pedro Miguel Pimentel ${ }^{\star}$ \\ University of the Azores \\ Business and Economics Department, CEEAplA,
}

R. Mãe de Deus, 9500 Ponta Delgada, Portugal, ppimentel@notes.uac.pt

\author{
José Azevedo-Pereira \\ ISEG \\ Universidade Técnica de Lisboa \\ Department of Management \\ R. Miguel Lupi, 1249-078 Lisboa, Portugal, jpereira@,iseg.utl.pt \\ Gualter Couto \\ University of the Azores, \\ Business and Economics Department, CEEAplA \\ R. Mãe de Deus, 9500 Ponta Delgada, Portugal, gcouto@notes.uac.pt
}

This draft: May 2007

\begin{abstract}
The present paper investigates the optimal timing of investment for a high speed rail (HSR) project, in an uncertain environment, using a real options analysis (ROA) framework. It develops a continuous time framework with stochastic demand that allows for the determination of the optimal timing of investment and the value of the option to defer in the overall valuation of the project. The modelling approach used is based on the differential utility provided to railway users by the HSR service.
\end{abstract}

Keywords: Real Options, Uncertainty, Timing, Waiting, Investment, High Speed Rail. JEL classification: D81, D83, D92.

\footnotetext{
* The research of the first author was supported by FCT grant SFRH/BD/23576/2005.
} 


\section{Introduction}

Today's global and dynamic business market is characterized by a growing uncertainty that affects significantly the decision-making processes within business organizations. In this sense, dealing with uncertainty and taking optimal decisions regarding investment opportunities becomes a way of achieving supremacy over competition.

Flexibility is crucial to perform efficiently, for instance, in terms of technological changes, competition's shifts, or even in order to limit potential losses related to unexpected adverse scenarios in the market.

Given the ineffectiveness of the traditional capital budgeting techniques in uncertain environments (Trigeorgis, 1996), the conceptual framework available in order to appraise complex investments in real assets consists in using real options analysis (ROA) techniques (Dixit and Pindyck, 1994).

ROA has changed the investment valuation paradigm, due to its ability to cope with decision makers' flexibility. In the offing period, new information may lead to a total or partial change of the initial plan, including the abandonment of the project.

In spite of having emerged in the academy, (Brennan and Schwartz, 1985; McDonald and Siegel, 1986; Dixit, 1989; Pindyck, 1991; and Dixit and Pindyck, 1994, amongst others), this new paradigm has already made an impact in the business world, since an increasing number of companies and managers are adopting a real options perspective. Especially in capital budgeting decisions and in the assessment of the corresponding strategic positioning and competitiveness (Paddock, Siegel and Smit, 1988; Nichols, 1994; Kallberg and Laurin, 1997; Moel and Tufano, 2002; Smit, 2003; etc.).

The modelling framework proposed in this paper is inspired by a set of projects for the development of high speed rail (HSR) lines in Europe. The structuring nature of the projects for the countries involved; the need to renew the railway sector; the huge amounts of money needed; the uncertainty about the timings to invest and the economic challenge inherent in developing a conceptual setting for a decision that needs to be taken in the interest of the entire set of European taxpayers, all play a part in providing relevance to the study of the embedded option to defer and the optimal timing to invest. 


\section{Real Options in Major Projects in the Transportation Sector}

Although usually linked to political discussion and controversy, transport infrastructures tend to be understood as critical for the sustainable growth and development of any economy. According to Wilson (1986), since 1870 economists have been drawing their attention towards the transport industry in general, and to the railway sector in particular. The same author suggests that wrong transportation policies and the corresponding investment mistakes in transport infrastructures may compromise seriously economic growth. To prevent this type of outcome, it is important to develop and apply suitable decision criteria based upon sound cost/benefit analysis.

Infrastructure investments that are usually understood to provide benefit and leverage to the economic growth of whole regions include investments in seaports, airports and railways links, energy networks, road systems, amongst others.

The size, budget and impact in the global economic activity lead big transportation investments to assume the role of strategic options. Almost all these investments include a portfolio of options intended to, at some extent, protect the enormous funds needed to implement the project from failure.

Rose (1998) has valued the concession of a toll road, considering the existence of two options interacting with each other. The author assumed that the traffic volume followed a geometric Brownian motion and used Monte Carlo simulation to compute $i$ ) the value of the embedded call option that allowed for the early acquisition of the project by the franchiser and ii) the option to defer regarding the payment of the corresponding fees' by the franchisee. Similarly, Brandão (2002) applied the Copeland and Antikarov's (2003) framework to value several options embedded in a project that included the building and operation of highways in Brazil.

More recently, two other empirical ROA works focused on the valuation of structural investments in the transportation sector, were published: Smit (2003) and Bowe and Lee (2004). The first, analyses the expansion of an airport, while the second is apparently pioneer in the analysis of a railway transportation project.

Investments in infrastructure or platform assets generate other investments opportunities that change the competitive standing of the companies involved. Smit (2003) combines ROA and game theory to capture the intrinsic value derived from the company's positioning adjustment inside the industry, with an empirical application to the expansion of a European airport. His work has helped to fill in a gap in the real 
options literature, where researchers have tended to, either, ignore competition, underestimating the impact of a competitive entry, or assume that the competition is exogenous to the valuation process. Smit (2003) has chosen to develop his work in a discrete time framework, arguing that it makes it more simple and available to management teams, at the same time that includes potential asymmetries amongst competitors and makes possible the definition of alternative stochastic processes.

The main contribution of Smit's (2003) work is due to the fact that he evaluates the growing opportunities generated by an infrastructure as a game of several sequential exercises. Following Trigeorgis' (1996) developments, Smit (2003) starts by valuing the project without expansion opportunities, using traditional capital budgeting decision techniques, and subsequently valued the embedded growth opportunities in a competitive context with other European airports.

Similarly to Smit (2003), Bowe and Lee (2004) apply binomial analysis. However, they use a logarithmic transformation similar to Trigeorgis (1991), to evaluate the high speed train project in Taiwan, comparing the obtained results with a valuation based on traditional capital budgeting decision techniques. The work embraces the valuation of three different options (expand, reduce and defer) and the according interactions, included in a project that does not pay dividends. Nevertheless, as stated by the authors, this type of analysis should incorporate the effect of dividends in order for the framework to become close enough to real life situations to deserve proper consideration by companies.

\section{Investment Valuation Using a Real Options Framework}

In a HSR project, at any moment in time, the owner of the investment's rights holds the possibility of acquiring the future cash flow generated by the venture, in exchange for the payment of the corresponding implementation costs. Thus, we are dealing with an option to invest.

Considering the investment in a HSR line as an optimal stopping problem allows us to determine the value of the embedded option to defer. Following, the work of McDonald and Siegel (1986) and Salahaldin and Granger (2005), it also permits to determine the optimal timing to invest. 
In the present paper it will be assumed not only that the option to defer is perpetual in nature $(T=\infty)$, but also that, once implemented, the investment will produce perpetual benefits. Without major technological changes, the impact of these assumptions in the global valuation should not be unreasonable for two reasons. In the first place, because the present value of the more remote cash flows tends naturally to zero. In the second place, because maintenance and conservation - whose expenses are taken into consideration - tend to restore the operational aptitude of the assets in place and the corresponding flow of benefits.

\subsection{Optimal Timing to Invest - Investment in one Period}

In a context of the nature above mentioned, a decision to implement a project in a non-optimal moment, implies destruction of value. Therefore, finding the optimal timing offers the possibility to study the impact of the ability to delay in the global value of the project.

Thus, it is important to answer the question of when to invest, or at least find a critical value that might support in a rational way the decision of implementing the investment. The irreversibility features of the investment, given that there is no other use for the project rather than the railways, emphasise the importance of estimating the optimal timing to invest.

The model proposed here draws on the work of Salahaldin and Granger (2005) on the valuation of sustainable systems of urban transport aimed at relieving air pollution. It is a model that comprises a unique change from an inactive to an active state, and considers a single stochastic variable.

Because investment in infrastructures, like HSR lines, will affect the economic and social conditions of future generations, it should be assessed considering a global point of view, in terms of economic welfare. In an uncertain environment, it will only make sense to invest in such a project, if the economic value of the utility provided by the resulting benefits is able to surpass the joint value of the option to defer (lost by investing) and of the utility provided by the conventional railway system to its users.

Investing, in a moment other than the corresponding optimal timing, implies a reduction in the global level of utility achieved by the users, compromising seriously the projects' success. In such circumstances, any potential user may always maintain his 
current level of utility, choosing to travel in the conventional railway line, rather than in the new HSR service. If a suboptimal investment timing is chosen, the ability of the HSR service to attract clients will be strongly distressed.

At any moment users can choose to travel in the conventional railway, without any constraints. Consequently, to maintain the users' utility, the fraction of the new investment supported by each one must be identical to the sum of the benefits earned resulting from the reduction of the travel time and the conventional service fare saved, net of variable and fixed operational costs upheld.

Given a fixed amount to invest, the higher the demand, the higher the expected net benefit per capita. Consequently, higher levels of demand tend to lead to the anticipation of the optimal invest timings. The main source of uncertainty derives obviously from the level of future demand for the HSR service.

We will consider that the demand for the new high speed service, $x_{t}$, follows a geometric Brownian motion process:

$$
\frac{d x_{t}}{x_{t}}=\mu d t+\sigma d w
$$

Similar assumptions may be found in Rose (1998), with the purpose of modelling highway traffic; in Salahaldin and Granger (2005), with the purpose of modelling the dynamics of a city' population; and in Marathe and Ryan (2005) and Pereira et al. (2006) with the purpose of modelling airline demand.

In equation (3.1) $\mu$ and $\sigma$ represent the growth rate and the standard deviation of the demand for the HSR service. We assume that both parameters are constant in time. The Wiener process, $w_{t}$, has zero mean and standard deviation $\sigma \sqrt{d t}$.

Under these circumstances, it is reasonable to expect that, in the future, the natural demand for HSR will reach a level capable of providing a rational reason to invest in such a project.

In order to model such a situation we are going to assume that each user will face a cost for railway travel between two cities, $\psi$, whose global worth will be a function of the value of time for the user, $\eta$, and the travel fare, $p$. According to the literature, both these variables exhibit a relationship to the global demand for railway services (vide Owen e Phillips, 1987; Wardman, 1994; and Wardman 1997). 
Considering the relationship between the value of travel time and the demand for faster railway services (Owen e Phillips, 1987; and Wardman, 1994), the following functional form will be used:

$$
\eta=\beta x^{\delta_{\beta}}
$$

In this functional form, $\delta_{\beta}$ represents the elasticity between the value of travel time $\eta$ and the HSR demand $x$. Consequently, $\beta$ is the scale parameter between demand, $x$, and the value of travel time, $\eta$, given by:

$$
\beta=\eta x^{-\delta_{\beta}}
$$

Concerning the relationship between the fare value and the demand for railway services, this will be given by the functional form (Owen e Phillips, 1987):

$$
p=\alpha x^{\delta_{\alpha}}
$$

The elasticity between the fare value, $p$, and the HSR demand, $x$, is represented by the parameter $\delta_{\alpha}$. The scale parameter $\alpha$, that relates demand and the fare value, $p$, is given by:

$$
\alpha=p x^{-\delta_{\alpha}}
$$

The demand may be inferred from the preferences of a risk neutral representative user, with a utility function $U=c$, in which $c$ represents the mean consumption of all users that constitute the overall demand. The budget constraint is given by $c+\psi=m$, in which $\psi$ represents the travel cost and $m$ the individual disposable income by unit of time. Analytically, we have:

$$
c=m-\psi
$$

Replacing the level of consumption in the utility function, will allow for the determination of the following indirect utility function, $V$, representative of the value that each user attributes to a railway trip:

$$
V=U=m-\psi
$$

The relationship between demand $x_{t}$ and value of travel time, $i$ ) in the period of time that precedes investment, $\eta_{0}$;ii) during the period of effective investment, $\eta_{1}$; and 
iii) after the investment's implementation, $\eta_{2}$, is represented, respectively, by $\beta_{0}, \beta_{1}$ and $\beta_{2}$. Since the new rail service will save travel time and, in consequence, will reduce the value of travel time from $\eta_{0}$ to $\eta_{2}$, it will be reasonable to expect that from the pre-investment period to the operational phase $\beta_{0}$ will change to $\beta_{2}$ with $\beta_{0}>\beta_{2}$. The difference between $\beta_{0}$ and $\beta_{2}$ reflects the decrease in travel time.

Meanwhile, for the moment, we will assume that the investment will take place during a single period of time. Thus, the relationship between demand and travel costs, during the construction period $\beta_{1}$ is assumed to be equal to $\beta_{2}$.

Analytically, the cost of travelling in a conventional railway, $\psi_{0}$, and the cost of travelling in HSR, $\psi_{2}$, will be represented by the following equations,

$$
\begin{gathered}
\psi_{0}=\beta_{0} x_{t}^{\delta_{\beta}}+\alpha_{0} x_{t}^{\delta_{\alpha}} \\
\psi_{2}=\beta_{2} x_{t}^{\delta_{\beta}}
\end{gathered}
$$

For modelling purposes, the conventional railway travel cost, $\psi_{0}$, includes both the value of the travel time lost and the fare paid. In contrast, the HSR travel cost function here considered, $\psi_{2}$, is not affected by the value of the corresponding fare, $p_{2}$, because the current valuation framework assumes implicitly that each user will bear his part of the investment expenditure plus the corresponding operating costs per user. In other words, a socially acceptable HSR service fare is already implicitly considered in the valuation framework. Consequently, it does not make sense to duplicate it.

The existing conventional railway service that charges a fare $p_{0}$, enables us to identify the relationship between HSR demand, $x_{t}$, and the price of a substitute service (Owen and Phillips, 1987; and Wardman, 1997) given by equation (3.4).

As long as the investment is not implemented, the indirect utility function will be given by:

$$
V_{0}=m-\beta_{0} x^{\delta_{\beta}}-\alpha_{0} x^{\delta_{\alpha}}
$$

After the investment is implemented, users will continue to face a (smaller) cost in terms of time spent. However, since the analysis performed here takes into consideration all costs and benefits induced by the project (including not only capital 
investment expenditure, but also all fixed and variable operating costs), the new indirect utility function will be given by:

$$
V_{2}=m-\beta_{2} x^{\delta_{\beta}}-\omega-\frac{\varphi}{x}-\frac{\rho \gamma}{x}
$$

with $\gamma$ representing the capital investment expenditure, $\rho$ the discount rate, $\omega$ the variable operating costs and $\varphi$ the fixed operating costs. Notice that $\frac{\varphi}{x}$ and $\frac{\rho \gamma}{x}$ represent the fixed operating costs and the investment expenditure per unit of time, for each user that integrates the global demand for the HSR service. We assume implicitly that the outcomes of the investment will last for an unlimited time horizon.

The purpose is to carry out the investment without changing the present utility function equilibrium. In order to achieve this outcome, it will be necessary to find the critical demand level for $x^{*}$, above which it will be optimal to invest.

Noting that, in these terms, the whole framework might be understood as an intergeneration welfare problem, as previously stated, we may use the objective function of Ramsey-Koopmans adopted by Salahaldin and Granger (2005). Analytically, we have:

$$
\sup _{x^{*}} E_{x_{0}}\left(\int_{0}^{\tau} x_{t} V_{0}(x) e^{-\rho t} d t+\int_{\tau}^{\infty} x_{t} V_{2}(x) e^{-\rho t} d t\right)
$$

Where,

$$
\begin{aligned}
\tau= & \begin{array}{l}
\text { Moment of time in which the optimal value is achieved by the first } \\
\text { time; }
\end{array} \\
V_{0}(x)= & \text { Indirect utility function per unit of time before investment } \\
& \text { implementation, given by the equation }(3.10) ; \\
V_{2}(x)= & \text { Indirect utility function per unit of time after investment } \\
& \text { implementation, given by the equation (3.11); } \\
x_{t}= & \text { Demand throughout time, given by the equation (3.1); and } \\
x_{0}= & \text { Estimated demand at present. }
\end{aligned}
$$

Aggregating the utility of all users that constitute the potential demand before and after the investment, and replacing $V_{0}$ and $V_{2}$ for the corresponding values in (3.10) and (3.11), we get: 


$$
\sup _{x^{*}} E_{x_{0}}\left(\int_{0}^{\tau} e^{-\rho t}\left[m x_{t}-\beta_{0} x_{t}^{\theta_{\beta}}-\alpha_{0} x_{t}^{\theta_{\alpha}}\right] d t+\int_{\tau}^{\infty} e^{-\rho t}\left[m x_{t}-\beta_{2} x_{t}^{\theta_{\beta}}-\omega x_{t}-\varphi-\rho \gamma\right] d t\right)
$$

with $\theta_{\beta}=1+\delta_{\beta}$ and $\theta_{\alpha}=1+\delta_{\alpha}$.

Applying the Markov propriety as in Oksendal (2003), we will get:

$$
E_{x}\left(\int_{\tau}^{\infty} e^{-\rho t}\left[m x_{t}-\beta_{0} x_{t}^{\theta_{\beta}}-\alpha_{0} x_{t}^{\theta_{\alpha}}\right] d t\right)=E_{x}\left(e^{-\rho \tau} E_{x^{*}}\left(\int_{0}^{\infty} e^{-\rho t}\left[m x_{t}-\beta_{0} x_{t}^{\theta_{\beta}}-\alpha_{0} x_{t}^{\theta_{\alpha}}\right] d t\right)\right)
$$

in the second element of (3.13), we obtain

$$
\begin{aligned}
& E_{x_{0}}\left(\int_{\tau}^{\infty} e^{-\rho t}\left[m x_{t}-\beta_{2} x_{t}^{\theta_{\beta}}-\omega x_{t}-\varphi-\rho \gamma\right] d t\right)= \\
& =E_{x_{0}}\left(e^{-\rho \tau} E_{x^{*}}\left(\int_{0}^{\infty} e^{-\rho t}\left[m x_{t}-\beta_{2} x_{t}-\omega x_{t}-\varphi-\rho \gamma\right] d t\right)\right)
\end{aligned}
$$

Replacing the second element of (3.13) by the RHS of (3.15) and additionally adding and subtracting the RHS of (3.14), results in the following objective function,

$$
\sup _{x^{*}}\left[\begin{array}{l}
E_{x_{0}}\left(\int_{0}^{\infty} e^{-\rho t}\left[m x_{t}-\beta_{0} x_{t}^{\theta_{\beta}}-\alpha_{0} x_{t}^{\theta_{\alpha}}\right] d t\right)+ \\
\left.+E_{x_{0}}\left(e^{-\rho \tau} E_{x^{*}}\left(\int_{0}^{\infty} e^{-\rho t}\left[\left(\beta_{0}-\beta_{2}\right) x_{t}^{\theta_{\beta}}+\alpha_{0} x_{t}^{\theta_{\alpha}}-\omega x_{t}-\varphi-\rho \gamma\right] d t\right)\right)\right]
\end{array}\right]
$$

Since the first component does not depend on $x^{*}$, the problem may be rewritten, in the following terms:

$$
\sup _{x^{*}} E_{x_{0}}\left[e^{-\rho \tau}\left(E_{x^{*}}\left(\int_{0}^{\infty} e^{-\rho t}\left[\left(\beta_{0}-\beta_{2}\right) x_{t}^{\theta_{\beta}}+\alpha_{0} x_{t}^{\theta_{\alpha}}-\omega x_{t}-\varphi-\rho \gamma\right] d t\right)\right)\right]
$$

This objective function maximizes the net gain provided by an investment in a HSR link, in terms of travel costs for the corresponding users.

Simplifying, we have: 


$$
\begin{aligned}
& E_{x^{*}}\left(\int_{0}^{\infty} e^{-\rho t}\left[\left(\beta_{0}-\beta_{2}\right) x_{t}^{\theta_{\beta}}+\alpha_{0} x_{t}^{\theta_{\alpha}}-\omega x_{t}-\varphi-\rho \gamma\right] d t\right)= \\
& =\int_{0}^{\infty} e^{-\rho t}\left[\left(\beta_{0}-\beta_{2}\right) E_{x}^{*}\left(x_{t}^{\theta_{\beta}}\right)+\alpha_{0} E_{x}^{*}\left(x_{t}^{\theta_{\alpha}}\right)-\omega E_{x}^{*}\left(x_{t}\right)-\varphi-\rho \gamma\right] d t
\end{aligned}
$$

We know that $x_{t}$ follows a geometric Brownian motion described by (3.1). Thus,

$$
E_{x}^{*}\left(x_{t}^{\theta}\right)=\left(x^{*}\right)^{\theta} e^{\left(\theta \mu_{x} t+\frac{1}{2} \theta(\theta-1) \sigma_{x}^{2} t\right)}
$$

The existence of a future optimal timing to invest requires the need to respect the following condition $\rho-\theta \mu_{x}-\frac{1}{2} \theta(\theta-1) \sigma_{x}^{2}>0$. This condition imposes the demand growth rate to be lower than discount rate, thus providing a rational economic interpretation to the underlying mathematical developments. Simplifying again and under this new condition, we have:

$$
\begin{aligned}
& \int_{0}^{\infty} e^{-\rho t}\left[\left(\beta_{0}-\beta_{2}\right) E_{x}^{*}\left(x_{t}^{\theta_{\beta}}\right)+\alpha_{0} E_{x}^{*}\left(x_{t}^{\theta_{\alpha}}\right)-\omega E_{x}^{*}\left(x_{t}\right)-\varphi-\rho \gamma\right] d t= \\
& =\frac{2\left(\beta_{0}-\beta_{2}\right)\left(x^{*}\right)^{\theta_{\beta}}}{2 \rho-2 \mu_{x} \theta_{\beta}-\theta_{\beta}^{2} \sigma_{x}^{2}+\theta_{\beta} \sigma_{x}^{2}}+\frac{2 \alpha_{0}\left(x^{*}\right)^{\theta_{\omega}}}{2 \rho-2 \mu_{x} \theta_{\alpha}-\theta_{\alpha}^{2} \sigma_{x}^{2}+\theta_{\alpha} \sigma_{x}^{2}}-\frac{\omega\left(x^{*}\right)}{\rho-\mu_{x}}-\frac{\varphi}{\rho}-\gamma
\end{aligned}
$$

Rewriting (3.17) taking into consideration the result (3.20), we achieve:

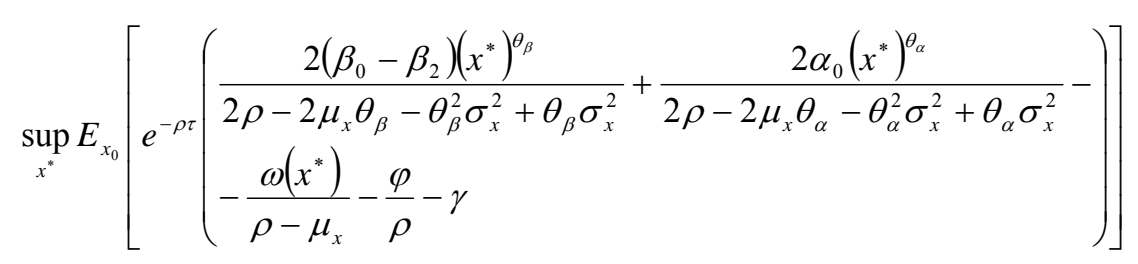

With,

$$
\begin{gathered}
A=\frac{2\left(\beta_{0}-\beta_{2}\right)}{2 \rho-2 \mu_{x} \theta_{\beta}-\theta_{\beta}^{2} \sigma_{x}^{2}+\theta_{\beta} \sigma_{x}^{2}} \\
B=\frac{2 \alpha_{0}}{2 \rho-2 \mu_{x} \theta_{\alpha}-\theta_{\alpha}^{2} \sigma_{x}^{2}+\theta_{\alpha} \sigma_{x}^{2}} \\
C=-\frac{\varphi}{\rho}
\end{gathered}
$$




$$
D=-\gamma
$$

and

$$
F=-\frac{\omega}{\rho-\mu_{x}}
$$

function (3.21) becomes,

$$
\sup _{x^{*}} E_{x_{0}}\left[e^{-\rho \tau}\left(A\left(x^{*}\right)^{\theta_{\beta}}+B\left(x^{*}\right)^{\theta_{\alpha}}+F\left(x^{*}\right)+C+D\right)\right]
$$

Given the current demand level, the value of the project, $v$, is determined through the maximization of the function:

$$
v\left(t_{0}, x_{0}\right)=E_{x_{0}}\left[e^{-\rho \tau}\left(A\left(x^{*}\right)^{\theta_{\beta}}+B\left(x^{*}\right)^{\theta_{\alpha}}+F\left(x^{*}\right)+C+D\right)\right]
$$

That satisfies the differential equation,

$$
\frac{1}{2} \sigma_{x}^{2} x_{0}^{2} \frac{\partial^{2} v}{\partial x_{0}^{2}}+\mu_{x} x_{0} \frac{\partial v}{\partial x_{0}}-\rho v=0, \text { for } x \neq x^{*}
$$

Resulting from the simplification of the following partial differential equation:

$$
\frac{1}{2} \sigma_{x}^{2} x_{0}^{2} \frac{\partial^{2} v}{\partial x_{0}^{2}}+\mu_{x} x_{0} \frac{\partial v}{\partial x_{0}}+\frac{\partial v}{\partial t}=0, \text { for } x \neq x^{*}
$$

Equation (3.29) satisfies the following conditions:

1. Initial condition:

$$
v(0)=0
$$

2. Value matching condition:

$$
v\left(t_{0}, x_{0}\right)=A x_{0}^{\theta_{\beta}}+B x_{0}^{\theta_{\alpha}}+F x_{0}+C+D, \text { with } x_{0}=x^{*}
$$

and,

3. Smooth-pasting condition:

$$
v^{\prime}\left(t_{0}, x_{0}\right)=\theta_{\beta} A x_{0}^{\theta_{\beta}-1}+\theta_{\omega} B x_{0}^{\theta_{\omega}-1}+F, \text { with } x_{0}=x^{*}
$$

To solve (3.29) we substitute $v\left(t_{0}, x_{0}\right)=e^{-\rho t_{0}} \Phi\left(x_{0}\right)$, where $\Phi$ represents the projects' value function at any moment in time. Therefore, (3.29) becomes, 


$$
\frac{1}{2} \sigma_{x}^{2} x_{0}^{2} \Phi^{\prime \prime}\left(x_{0}\right)+\mu_{x} x_{0} \Phi^{\prime}\left(x_{0}\right)-\rho \Phi\left(x_{0}\right)=0, \text { for } x \neq x^{*}
$$

With the following conditions:

1. Initial condition:

$$
\Phi(0)=0
$$

2. Value matching condition:

$$
\Phi\left(x_{0}\right)=A x_{0}^{\theta_{\beta}}+B x_{0}^{\theta_{\alpha}}+F x_{0}+C+D, \text { with } x_{0}=x^{*}
$$

and,

3. Smooth-pasting condition

$$
\Phi^{\prime}\left(x_{0}\right)=\theta_{\beta} A x_{0}^{\theta_{\beta}-1}+\theta_{\alpha} B x_{0}^{\theta_{\alpha}-1}+F, \text { with } x_{0}=x^{*}
$$

Since equation (3.34) is a Cauchy-Euler second order homogeneous differential equation, the solution may be written as,

$$
\Phi\left(x_{0}\right)=a_{1} x_{0}^{r_{1}}+a_{2} x_{0}^{r_{2}}
$$

where $r_{1}$ and $r_{2}$ are the two roots of the quadratic equation:

$$
\frac{1}{2} \sigma_{x}^{2} r(r-1)+\mu_{x} x_{0} r-\rho=0
$$

given by,

$$
r_{1}=\frac{\left(\frac{1}{2} \sigma_{x}^{2}-\mu_{x}\right)+\sqrt{\left(\mu_{x}-\frac{1}{2} \sigma_{x}^{2}\right)^{2}+2 \rho \sigma_{x}^{2}}}{\sigma_{x}^{2}}
$$

and

$$
r_{2}=\frac{\left(\frac{1}{2} \sigma_{x}^{2}-\mu_{x}\right)-\sqrt{\left(\mu_{x}-\frac{1}{2} \sigma_{x}^{2}\right)^{2}+2 \rho \sigma_{x}^{2}}}{\sigma_{x}^{2}}
$$

As $a_{2} x_{0}^{r_{2}}$ tends to the infinity when $x_{0}$ tends to zero, according to the initial condition (3.35) and $\Phi\left(x_{0}\right)$ needs to be limited when $x_{0} \rightarrow 0, a_{2}=0$. Thus, equation (3.38) becomes, 


$$
\Phi\left(x_{0}\right)=a_{1} x_{0}^{r_{1}}
$$

Using the condition $\Phi\left(x^{*}\right)=A x^{* \theta_{\beta}}+B x^{* \theta_{\alpha}}+F x^{*}+C+D$ that results from the substitution of $x_{0}$ by $x^{*}$ in equation (3.36), we find the coefficient $a_{1}=A x^{* \theta_{\beta}-r_{1}}+B x^{* \theta_{\alpha}-r_{1}}+F x^{* 1-r_{1}}+C x^{*-r_{1}}+D x^{*-r_{1}}$, concluding that the solution of (3.29) is,

$$
v\left(t_{0}, x_{0}\right)=e^{-\rho t_{0}}\left[A x^{* \theta_{\beta}-r_{1}}+B x^{* \theta_{\alpha}-r_{1}}+F x^{* 1-r_{1}}+C x^{*-r_{1}}+D x^{*-r_{1}}\right] x_{0}^{r_{1}}
$$

For a given value of $x_{0}$ in $t_{0}=0$, the value of $x^{*}$ that maximizes $v\left(0, x_{0}\right)$ is implicitly given by the equation:

$$
A x^{* \theta_{\beta}-r_{1}}\left(\theta_{\beta}-r_{1}\right)+B x^{* \theta_{\omega}-r_{1}}\left(\theta_{\alpha}-r_{1}\right)+F x^{* 1-r_{1}}\left(1-r_{1}\right)-C x^{-r_{1}} r_{1}-D x^{-r_{1}} r_{1}=0
$$

The critical value $x^{*}$ can only be found through numerical solution of (3.44), except if two assumptions are made. The first assumption related to equality between the HSR demand/value of travel time elasticity and the HSR demand/conventional service fare cross elasticity, conducting to $\theta_{\beta}=\theta_{\alpha}=\theta$. The second assumption comes from the possibility of neglecting the operational variable costs, $F=0$, considering the operational characteristics of the project. Taking these two conditions into account, $x^{*}$ has the following closed form solution:

$$
x^{*}=\exp \left[\frac{\ln \frac{-r_{1}(C+D)}{(A+B)\left(r_{1}-\theta\right)}}{\theta}\right]
$$

The critical value $x^{*}$ represents the level of demand that, when reached, justifies (turns optimal) an immediate implementation of the project.

This solution preserves utility equilibrium between HSR and conventional service for railway users, making the optimal solution independent of the original income $\mathrm{m}$ and the initial level of demand for the HSR service $x_{0}$. The fact that the whole framework is aimed at achieving a better level of global economic welfare, based on the equilibrium between the utility of two similar services, turns this model especially adequate to analyse governmental scale investment decisions. 


\subsection{Optimal Timing to Invest - Investment over Several Periods}

Large projects normally take time to implement. Thus, it is crucial to include this feature in the ROA's model, allowing the time-to-build effect to be incorporated.

Relaxing the assumption previously made at this level and allowing $\beta_{1} \neq \beta_{2}$, we create a transition period that corresponds to the time needed to build the HSR link.

A new HSR link can only start to operate after all the inherent engineering and development work is finished. Consequently, during this building period $n$, the cost of travelling is still given by $\psi_{0}$, so $\beta_{1}$ remains equal to $\beta_{0}\left(\beta_{1}=\beta_{0}\right)$. When the HSR starts to operate, the cost of travelling will change to $\psi_{2}$, with $\beta_{2}$ incorporating the decrease in travel time.

The new Ramsey-Koopmans objective function becomes,

$$
\sup _{x^{*}} E_{x_{0}}\left(\int_{0}^{\tau} X_{t+n} e^{-\rho n} V_{0}\left(x_{t+n}\right) e^{-\rho t} d t+\int_{\tau}^{\infty} X_{t+n} e^{-\rho n} V_{2}\left(x_{t+n}\right) e^{-\rho t} d t\right)
$$

Where, now:

$$
\begin{aligned}
V_{0}\left(x_{t+n}\right)= & \text { Indirect utility function by unit of time before the beginning of the } \\
& \text { HSR operation; } \\
V_{2}\left(x_{t+n}\right)= & \text { Indirect utility function by unit of time after the beginning of the } \\
& \text { HSR operation; } \\
n= & \text { Time-to-build (construction) of the investment; }
\end{aligned}
$$

With,

$$
V_{0}\left(x_{t+n}\right)=m_{t+n}-\beta_{0} x_{t+n}^{\delta_{\beta}}-\alpha_{0} x_{t+n}^{\delta_{\alpha}}
$$

and

$$
V_{2}\left(x_{t+n}\right)=m_{t+n}-\beta_{2} x_{t+n}^{\delta_{\beta}}-\omega-\frac{\varphi}{x_{t+n}}-\frac{\rho \gamma e^{\rho n}}{x_{t+n}}
$$

Considering the global utility of all the users that constitute the demand before and after the HSR link starts to operate, and substituting $V_{0}$ and $V_{2}$ from (3.47) and (3.48) into (3.46), we obtain:

$$
\sup _{x^{*}} E_{x_{0}}\left(\begin{array}{l}
\int_{0}^{\tau} e^{-\rho t}\left[\left(m_{t+n} x_{t+n}-\beta_{0} x_{t+n}^{\theta_{\beta}}-\alpha_{0} x_{t+n}^{\theta_{\alpha}}\right) e^{-\rho n}\right] d t+ \\
+\int_{\tau}^{\infty} e^{-\rho t}\left[\left(m_{t+n} x_{t+n}-\beta_{2} x_{t+n}^{\theta_{\beta}}\right) e^{-\rho n}-\omega x_{t+n} e^{-\rho n}-\varphi e^{-\rho n}-\rho \gamma\right] d t
\end{array}\right)
$$


with $\theta_{\beta}=1+\delta_{\beta}$ and $\theta_{\alpha}=1+\delta_{\alpha}$.

Using again the Markov propriety from Oksendal (2003), with a simplification identical to that performed in the previous section, it is possible to obtain the following objective function,

$$
\sup _{x^{*}}\left[\begin{array}{l}
E_{x_{0}}\left[\int_{0}^{\infty} e^{-\rho t}\left[\left(m_{t+n} x_{t+n}-\beta_{0} x_{t+n}^{\theta_{\beta}}-\alpha_{0} x_{t+n}^{\theta_{\alpha}}\right) e^{-\rho n}\right] d t\right]+ \\
+E_{x_{0}}\left[e^{-\rho \tau} E_{x^{*}}\left(\int_{0}^{\infty} e^{-\rho t}\left[\left(\beta_{0}-\beta_{2}\right) x_{t+n}^{\theta_{\beta}} e^{-\rho n}+\alpha_{0} x_{t+n}^{\theta_{\alpha}}-\omega x_{t+n} e^{-\rho n}-\varphi e^{-\rho n}-\rho \gamma\right] d t\right)\right]
\end{array}\right]
$$

The first element does not depend on $x^{*}$, so (3.50) may be rewritten in the following terms:

$$
\sup _{x^{*}} E_{x_{0}}\left[e^{-\rho \tau}\left[E_{x^{*}}\left[\int_{0}^{\infty} e^{-\rho t}\left[\left(\beta_{0}-\beta_{2}\right) x_{t+n}^{\theta_{\beta}} e^{-\rho n}+\alpha_{0} x_{t+n}^{\theta_{\alpha}}-\omega x_{t+n} e^{-\rho n}-\varphi e^{-\rho n}-\rho \gamma\right] d t\right]\right]\right]
$$

This objective function, similar to equation (3.17), maximizes the net utility gain, provided by an investment in a HSR link, in terms of travel costs for the corresponding users. In contrast to (3.17), this new formulation considers that after the decision to implement the project a $n$ building period will need to take place, before the HSR link may start to operate.

Simplifying, we have,

$$
\begin{aligned}
& E_{x^{*}}\left(\int_{0}^{\infty} e^{-\rho t}\left[\left(\beta_{0}-\beta_{2}\right) x_{t+n}^{\theta_{\beta}} e^{-\rho n}+\alpha_{0} x_{t+n}^{\theta_{\alpha}} e^{-\rho n}-\omega x_{t+n} e^{-\rho n}-\varphi e^{-\rho n}-\rho \gamma\right] d t\right)= \\
& =\int_{0}^{\infty} e^{-\rho t}\left[\left(\beta_{0}-\beta_{2}\right) E_{x}^{*}\left(x_{t+n}^{\theta_{\beta}}\right) e^{-\rho n}+\alpha_{0} E_{x}^{*}\left(x_{t+n}^{\theta_{\alpha}}\right) e^{-\rho n}-\omega E_{x}^{*}\left(x_{t+n}\right) e^{-\rho n}-\varphi e^{-\rho n}-\rho \gamma\right] d t
\end{aligned}
$$

Knowing that $x_{t}$ follows a geometric Brownian and that $E_{x}^{*}\left(x_{t}^{\theta}\right)$ is given by (3.19), then,

$$
E_{x}^{*}\left(x_{t+n}^{\theta}\right)=\left(x^{*}\right)^{\theta} e^{\left(\theta \mu_{x}+\frac{1}{2} \theta(\theta-1) \sigma_{x}^{2}\right)(t+n)}
$$

Simplifying again and under the condition that $\rho-\mu_{x} \theta-\frac{1}{2} \theta(\theta-1) \sigma_{x}^{2}>0$, we have, 


$$
\begin{aligned}
& \int_{0}^{\infty} e^{-\rho t}\left[\left(\beta_{0}-\beta_{2}\right) E_{x}^{*}\left(x_{t+n}^{\theta_{\beta}}\right) e^{-\rho n}-\omega E_{x}^{*}\left(x_{t+n}^{\theta_{\omega}}\right) e^{-\rho n}-\varphi e^{-\rho n}-\rho \gamma\right] d t= \\
& \frac{2\left(\beta_{0}-\beta_{2}\right)\left(x^{*}\right)^{\theta_{\beta}} e^{\left(\mu_{x} \theta_{\beta}+\frac{1}{2} \theta_{\beta}\left(\theta_{\beta}-1\right) \sigma_{x}^{2}\right) n} e^{-\rho n}}{2 \rho-2 \mu_{x} \theta_{\beta}-\theta_{\beta}^{2} \sigma_{x}^{2}+\theta_{\beta} \sigma_{x}^{2}}+\frac{2 \alpha_{0}\left(x^{*}\right)^{\theta_{\alpha}} e^{\left(\mu_{x} \theta_{\alpha}+\frac{1}{2} \theta_{\alpha}\left(\theta_{\alpha}-1\right) \sigma_{x}^{2}\right) n} e^{-\rho n}}{2 \rho-2 \mu_{x} \theta_{\alpha}-\theta_{\alpha}^{2} \sigma_{x}^{2}+\theta_{\alpha} \sigma_{x}^{2}}- \\
& -\frac{\omega\left(x^{*}\right) e^{\left(\mu_{x}-\rho\right) n}}{\rho-\mu_{x}}-\frac{\varphi e^{-\rho n}}{\rho}-\gamma
\end{aligned}
$$

Rewriting (3.51) considering these simplifications, we get:

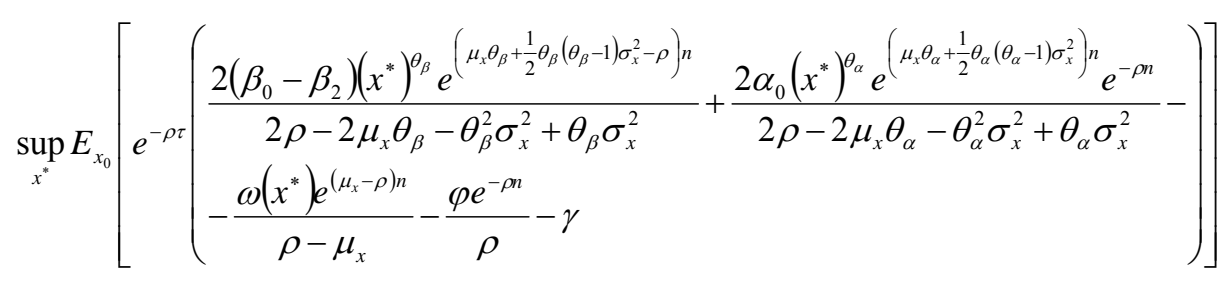

Now with,

$$
\begin{gathered}
A_{t c}=\frac{2\left(\beta_{0}-\beta_{2}\right) e^{\left(\mu_{x} \theta_{\beta}+\frac{1}{2} \theta_{\beta}\left(\theta_{\beta}-1\right) \sigma_{x}^{2}-\rho\right) n}}{2 \rho-2 \mu_{x} \theta_{\beta}-\theta_{\beta}^{2} \sigma_{x}^{2}+\theta_{\beta} \sigma_{x}^{2}} \\
B_{t c}=+\frac{2 \alpha_{0} e^{\left(\mu_{x} \theta_{\alpha}+\frac{1}{2} \theta_{\alpha}\left(\theta_{\alpha}-1\right) \sigma_{x}^{2}\right)} e^{-\rho n}}{2 \rho-2 \mu_{x} \theta_{\alpha}-\theta_{\alpha}^{2} \sigma_{x}^{2}+\theta_{\alpha} \sigma_{x}^{2}} \\
C_{t c}=-\frac{\varphi e^{-\rho n}}{\rho} \\
F_{t c}=-\frac{\omega e^{\left(\mu_{x}-\rho\right) n}}{\rho-\mu_{x}}
\end{gathered}
$$

and $D$ equal to (3.25). The subscript tc used above refers to solutions for $A, B, C$ and $F$ that apply to situations in which a time-to-build effect is considered.

The function that has to be maximized is similar to (3.28), with the inclusion of the above-mentioned difference in terms of notation:

$$
v\left(t_{0}, x_{0}\right)=E_{x_{0}}\left[e^{-\rho \tau}\left(A_{t c}\left(x^{*}\right)^{\theta_{\beta}}+B_{t c}\left(x^{*}\right)^{\theta_{\alpha}}+F_{t c}\left(x^{*}\right)+C_{t c}+D\right)\right]
$$


It is solved in the same way, since it satisfies the same differential equation (3.29) and also boundary conditions (3.31), (3.32) and (3.33).

For a given value of $x_{0}$ in $t=0$, the value of $x^{*}$ that maximizes $v\left(0, x_{0}\right)$ is given by the numerical solution of the equation:

$$
A_{t c} X^{* \theta_{\beta}-r_{1}}\left(\theta_{\beta}-r_{1}\right)+B_{t c} X^{* \theta_{\alpha}-r_{1}}\left(\theta_{\alpha}-r_{1}\right)+F_{t c}{ }^{*{ }_{1}-r_{1}}\left(1-r_{1}\right)-C_{t c} X^{-r_{1}} r_{1}-D x^{-r_{1}} r_{1}=0
$$

with $r_{1}$ given by (3.40).

When $\theta_{\beta}=\theta_{\alpha}=\theta$ and $F_{t c}=0, x^{*}$ is given by the following closed form solution similar to $(3.45)$ :

$$
x^{*}=\exp \left[\frac{\ln \frac{-r_{1}\left(C_{t c}+D\right)}{\left(A_{t c}+B_{t c}\right)\left(r_{1}-\theta\right)}}{\theta}\right]
$$

In this case, the critical value $x^{*}$ represents the level of demand that, when reached, justifies (turns optimal), an immediate implementation of a project whose HSR link will start to operate $n$ periods afterwards.

Using the traditional capital budgeting analysis, based on the concept of net present value (NPV), the rationale for taking the decision would be structurally similar, except that the decision would not be taken in an uncertain framework: the capital investment should only take place when the reduction in the cost of travelling provided by the HSR link and measured by the difference between $\psi_{0}$ and $\psi_{2}$ was enough to cover for the investment capital expenditure plus the operating costs. Analytically, for $\theta=\theta_{\beta}=\theta_{\omega}$, $F_{t c}=0$ and any $n \geq 0$, we have,

$$
\beta_{0} x_{t+n}^{\theta}+\alpha_{0} x_{t+n}^{\theta}>\beta_{2} x_{t+n}^{\theta}+\varphi+\rho \gamma e^{\rho n}
$$

Considering $x_{t+n}^{\theta} \equiv x_{t}^{\theta} e^{\theta_{\mu n}}$, it would only become optimal to invest if the demand level reached,

$$
x_{t}>\hat{x}=\left[\frac{\varphi+\rho \gamma e^{\rho n}}{\left(\beta_{0}-\beta_{2}-\alpha_{0}\right) e^{\theta_{\ell t}}}\right]^{\frac{1}{\theta}}
$$


with $\hat{x}$ representing the traditional capital budgeting analysis critical demand level, that once reached would justify the investment.

The comparison between the optimal rule of investment given by ROA (3.45) and by traditional capital budgeting analysis becomes evident if in an investment implemented in one single period of time, we consider $\theta=1$ as well as inexistence of fixed and variable costs $(\varphi=\omega=0)$.

In this case, equations (3.62) and (3.64) would become,

$$
\begin{gathered}
x^{*}=\frac{-r_{1} D}{\left(r_{1}-1\right)\left(A_{t c}+B_{t c}\right)} \\
\hat{x}=\frac{\rho \gamma}{\left(\beta_{0}-\beta_{2}+\alpha_{0}\right)}
\end{gathered}
$$

Equations (3.65) and (3.66) show that $x^{*}>\hat{x}$. Thus, when $\hat{x}<x_{t}<x^{*}$ a decision to implement based on a traditional capital budgeting analysis framework results in a value reduction for the whole project. In this situation, the value of the projects will be smaller than the sum of the capital expenditure and the value of the (sacrificed) option to defer. The ability to delay has value because it allows for uncertainty resolution.

\subsection{Valuation of an HSR Investment Using ROA Framework}

Considering the investment value function given by the (3.43), for a given level of $x_{0}$, with $t_{0}=0$, the value of an investment opportunity when $x_{0}<x^{*}$ is given by:

$$
v\left(x_{0}\right)=\left(\frac{x_{0}}{x^{*}}\right)^{r_{1}}\left[A_{t c} x^{* \theta_{\beta}}+B_{t c} x^{* \theta_{\alpha}}+F_{t c} x^{*}+C_{t c}+D\right]
$$

while for $x_{0} \geq x^{*}$ the value of the investment opportunity is given by:

$$
v\left(x_{0}\right)=\left[A_{t c} x^{\theta_{\beta}-r_{1}}+B_{t c} x^{\theta_{\alpha}-r_{1}}+F_{t c} x^{1-r_{1}}+C_{t c} x^{-r_{1}}+D x^{-r_{1}}\right] x_{0}^{r_{1}}
$$

Assuming $\theta=\theta_{\beta}=\theta_{\alpha}$ and $F_{t c}=0$, we may replace the critical value, $x^{*}$, given by (3.45) in the second part of the RHS of equation (3.67) and simplifying, the solution of the project's value function may be rewritten in the following terms: 


$$
v\left(x_{0}\right)= \begin{cases}\left(\frac{x_{0}}{x^{*}}\right)^{r_{1}}\left[\frac{\theta\left(C_{t c}+D\right)}{\theta-r_{1}}\right] & \text { for } x_{0}<x^{*} \\ \left(A_{t c}+B_{t c}\right) x_{0}^{\theta}+C_{t c}+D & \text { for } x_{0} \geq x^{*}\end{cases}
$$

with $C_{t c}, D, r_{1}, A_{t c}$ and $B_{t c}$ given by (3.58), (3.25), (3.40), (3.56) and (3.57).

In accordance to the literature (vide McDonald and Siegel, 1986; and Dixit and Pindyck, 1994), from the moment $\tau$, in which the optimal number of passengers is reached, $x^{*}$, the value of the option to defer is zero, since it is always better to implement the investment and receive in exchange the NPV - given by $A_{t c} x_{0}^{\theta_{\beta}}+B_{t c} x_{0}^{\theta_{\alpha}}+F_{t c} x_{0}+C_{t c}+D$ - of the expected decrease in the cost of travelling.

As long as the optimal timing to implement the investment is not reached, $t<\tau$, there is always an inherent value of waiting for new information about demand. In this case, the value of the option to defer is given by the difference between $v\left(x_{0}\right)$ and the NPV calculated using the expected demand in that moment.

In addition for allowing the inclusion of the impacts produced by $i$ ) the building period, ii) the fixed operating costs and iii) the variable operating costs, in the global value of the project, these developments take into consideration the elasticity between the value of travel time and demand. As the model is developed in terms of differential utility, factors other than those related to the cost of travelling (e.g., income), are assumed constant and do not influence the final outcome.

Whenever the elasticity between demand and value of travel time is null ( $\delta_{\beta}=0 \Rightarrow \theta_{\beta}=1$ ), we are implicitly assuming that, both, the conventional railway service and HSR service will not suffer real changes in terms value of travel time. Real changes in both services' fares imply positive levels of elasticity. Similarly, the conventional railway service fare remains constant in real terms whenever $\delta_{\alpha}=0 \Rightarrow \theta_{\alpha}=1$.

If $\theta_{\beta}>1$, increases in the value of travel time will be directly related to the passengers' growth rate. This type of demand behaviour for a faster rail transportation related to the value of travel time, besides being economically rational, is supported by the work of Owen and Phillips (1987) and Wardman (1994). In this sense it is 
acceptable that increases in the demand for the HSR service are, at least partially due to raises by the users in the value of travel time.

When $\theta_{\alpha}>1$ the cross elasticity between conventional railway fare and HSR demand is positive. Supported by the works of Owen and Phillips (1987) and Wardman (1997), increases in the fare of substitute service justify increases in the railway service demand.

The global value of a project determined by this ROA framework includes the economic worth of the ability to wait for uncertainty resolution, provided by the option to defer. When the ability to delay does not exist, as in the traditional capital budgeting decision analysis, this component is not taken in consideration and the global result will underestimate the corresponding true value. The value embedded in the option to postpone the investment derives from the incorporation of the value inherent in the "good tail" of the uncertainty regarding the demand by the HSR service. Turning parallel, the "bad tail" of demand uncertainty is limited by the option to carry on deferring (not investing), if the situation does not look attractive enough (McDonald and Siegel, 1986 and Dixit and Pindyck, 1994).

\section{Numerical Example}

Table 1. Base-case parameters for the project

\begin{tabular}{l|c}
\hline \multicolumn{1}{c|}{ Parameter } & Value \\
\hline$x_{0}-$ HSR demand at actual moment & $3 \mathrm{M}$ \\
$\gamma$ - Present value of the investment expenditures & $5,000 \mathrm{M} €$ \\
$\eta_{0}-$ Value of travel time in conventional railway service & $30 €$ \\
$\eta_{2}$ - Value of travel time in HSR service & $10 €$ \\
$p_{0}$ - Conventional railway service fare & $25 €$ \\
$\omega$ - Variable operating costs & $1 €$ \\
$\varphi$ - Fixed operating costs & $90 \mathrm{M} €$ \\
$\rho-$ Discount rate & 0.09 \\
$\mu_{x}-$ Expected growth rate of $x$ & 0.035 \\
$\sigma_{x}-$ Standard deviation of $x$ & 0.20 \\
$n-$ Number of years for the construction & 5 \\
$\delta_{\beta}-$ Elasticity between $x$ and $\eta$ & 0.60 \\
$\delta_{\alpha}-$ Cross elasticity between $x$ and $p_{0}$ & 0.40 \\
\hline Note: M Millions &
\end{tabular}


We are going to assume a project for the construction of a HSR link connecting two cities. The basic parameters are in Table 1. The conventional railway service operates in the same link. The new HSR service will reduce the travel time to $1 / 3$ comparatively to the conventional railway service.

Table 2 presents the HSR line investment valuation results for the base-case parameters.

Table 2. Project valuation results

\begin{tabular}{lr}
\hline \multicolumn{1}{c}{ Output } & \multicolumn{1}{c}{ Value } \\
\hline$x^{*}-$ Critical demand for HSR service $\left(\mathrm{n}^{\circ}{ }^{\mathrm{p}}\right.$ passengers $)$ & $10.777 \mathrm{M}$ \\
$v(x)-$ Investment Opportunity Value & $3,743.3 \mathrm{M} €$ \\
$n p v-$ Net Present Value & $254.2 \mathrm{M} €$ \\
\hline vod - Value of the Option do Defer & $3,489.1 \mathrm{M} €$ \\
\hline
\end{tabular}

Based on the results obtained, the construction of the HSR line should only start when the demand reaches 10,777 millions of passengers. Although the project registers a slightly positive NPV, shouldn't be implemented at the current time, concerning the uncertainty regarding the number of passengers of the new service. Maintaining "alive" this investment's opportunity has a value of 3,743 millions of euros, of which 93,21\% results from the value of the option to defer the investment.

Figure 1. Investment's opportunity value, NPV and value of the option to defer, for the base case

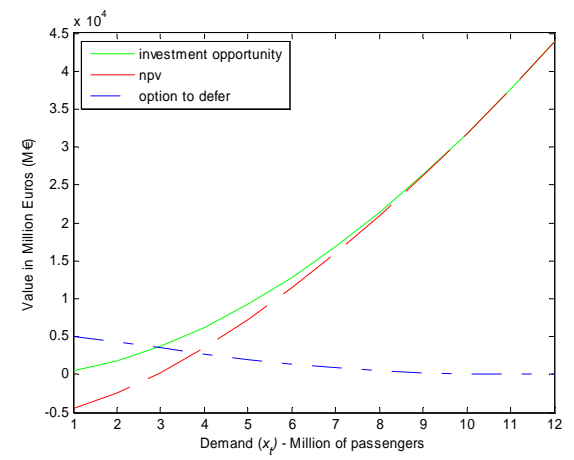

Figure 1 represents the evolution of the investment's opportunity value, the NPV and the option to defer according to the demand $x_{t}$ increase throughout time. As we may observe, for levels of demand higher than the critical demand level of 10,777 millions passengers, the option to defer the implementation no longer has value. Thus, 
from this point on, the decision to immediately implement the project is the one which maximizes the value for its owners.

Figure 2 to Figure 7 show the sensibility of the valuation indicators of the project regarding the variation of some parameters. Thus, we may notice that critical demand level $x^{*}$ varies inversely with the demand growth rate $\mu_{x}$ (Figure 2 ) and with the reduction of the value of travel time given by $\frac{\eta_{0}-\eta_{2}}{\eta_{0}}$ which the HSR line enables (Figure 7). For higher demand growth rates $\mu_{x}$ and with major reductions in the value of travel time, the present value of the benefits resulting from the project increases, justifying anticipating its implementation.

Figure 2. The impact of the growth rate
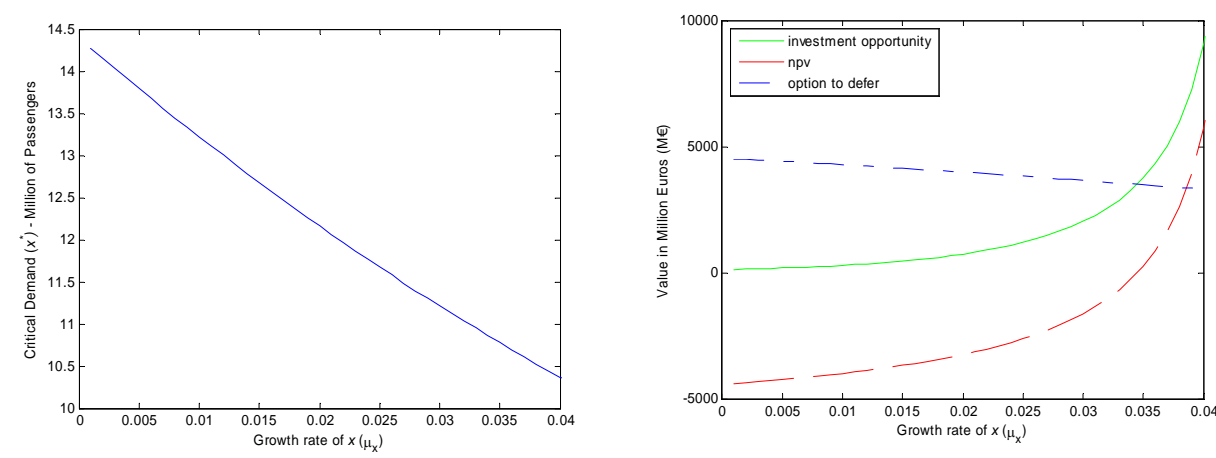

Figure 3. The impact of the discount rate
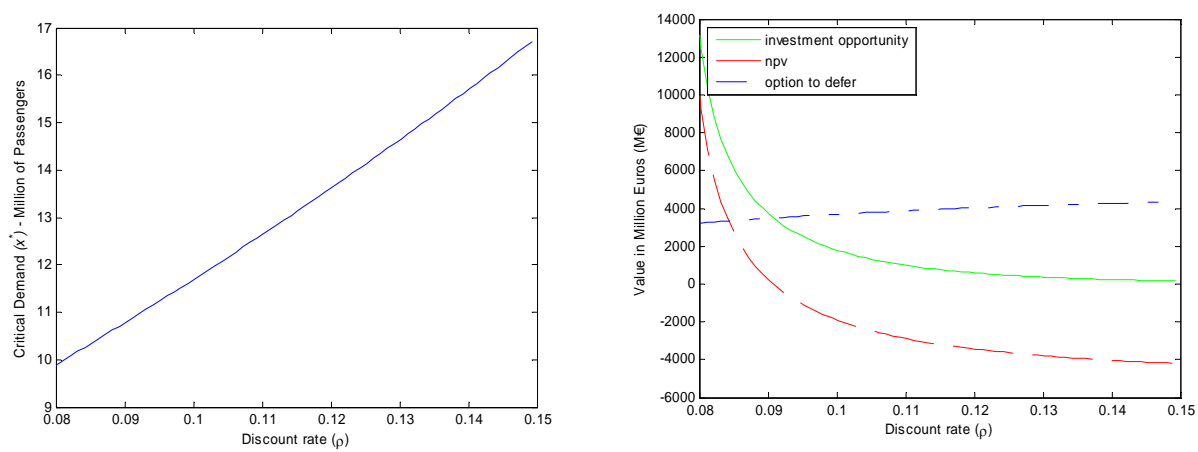
Figure 4. The impact of the investment expenditures
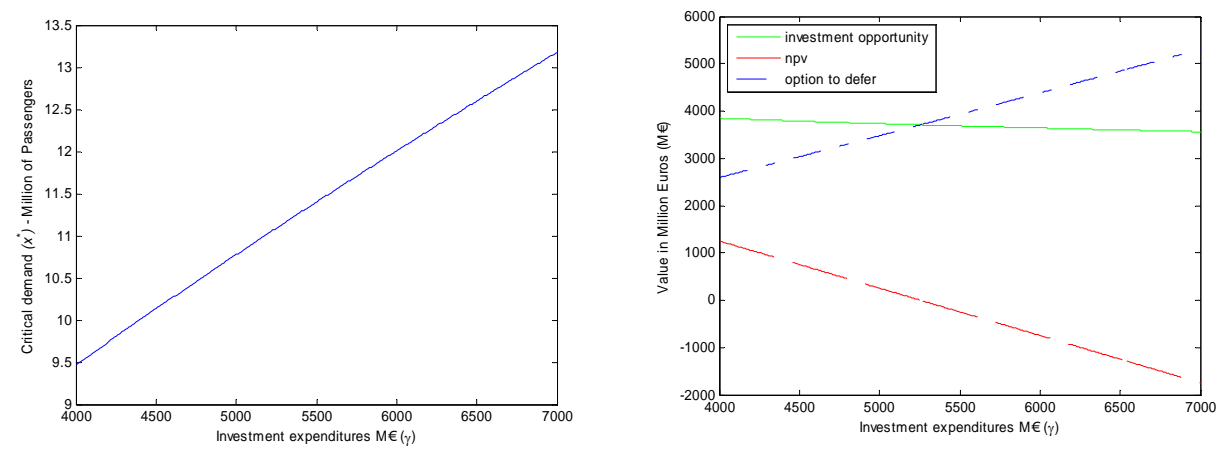

Figure 5. The impact of the volatility of the number of passengers
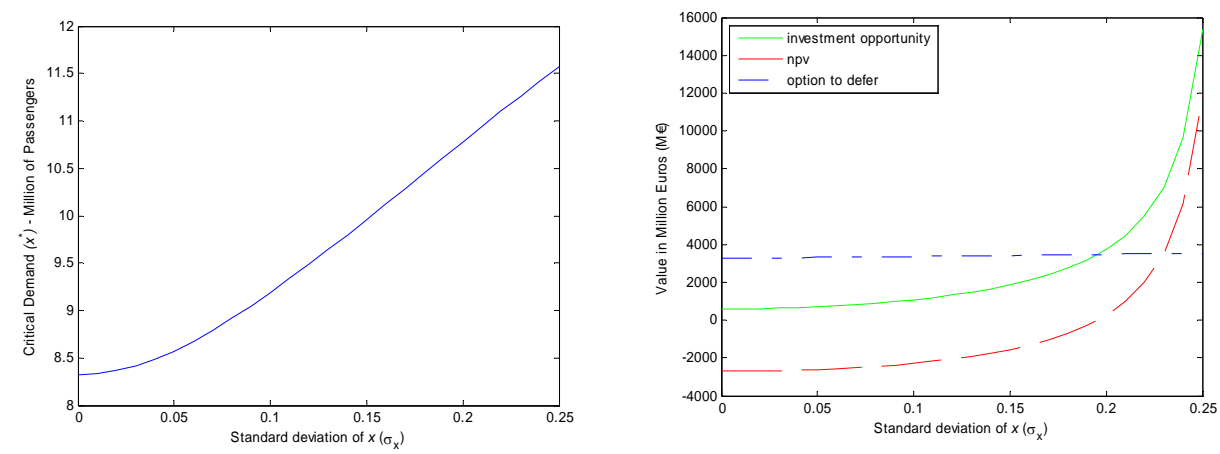

Figure 6. The impact of the time-to-build
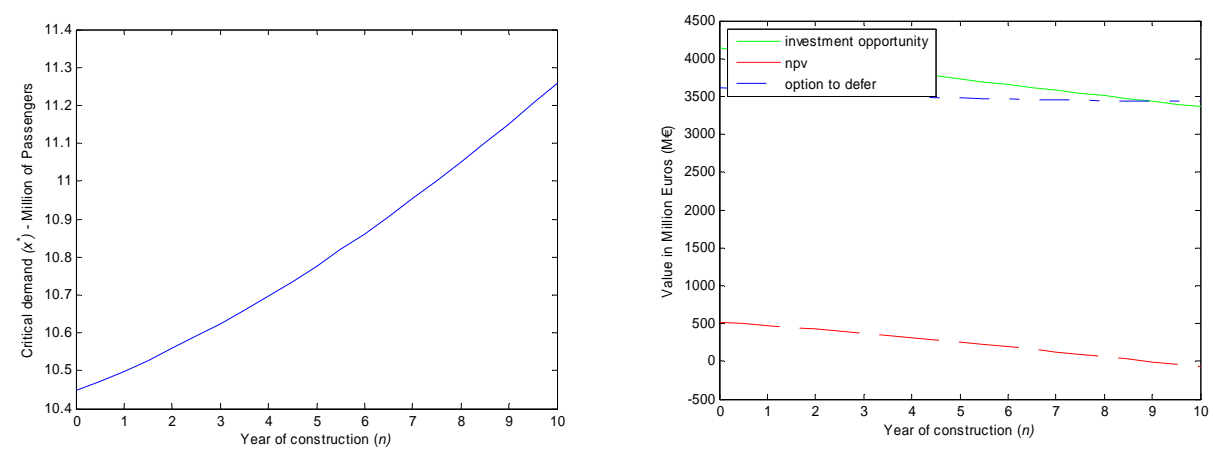

The other parameters analyzed assume a direct relationship with the critical level of demand $x^{*}$. Larger discount rates (Figure 3), larger investment's expenditures (Figure 4), larger volatility in the number of passengers (Figure 5) or more construction time needed (Figure 6) instigate significant postponements in the projects' implementation. 
Figure 7. The impact of the reduction in the value of travel time
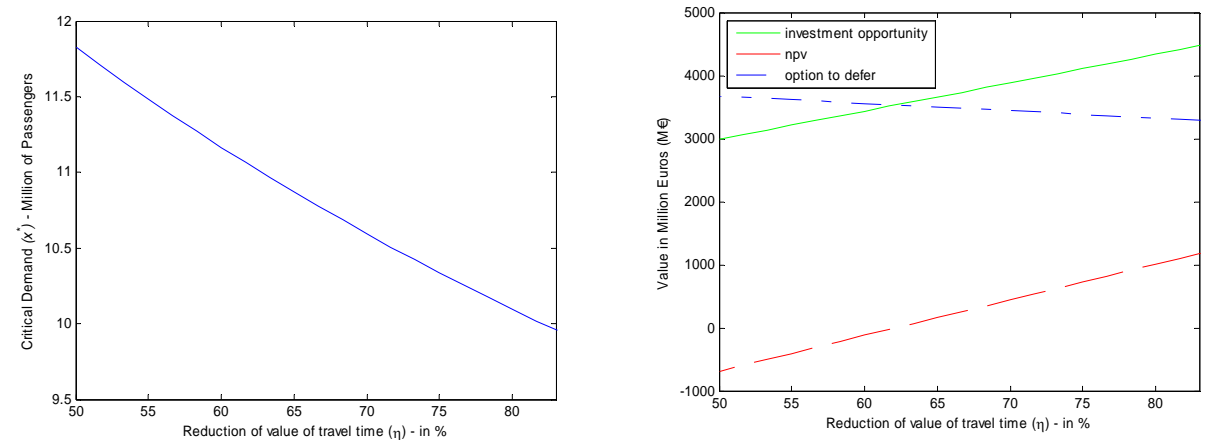

In presence of variations in any of the analyzed parameters, the investment's opportunity value and the NPV always register the same trend, for each one of the parameters, although with different drifts. It is worth noting the behaviour of NPV regarding increments in volatility. Figure 5 shows that NPV increases with uncertainty increase. This result originates from the fact that the valuation model incorporates the elasticity between HSR demand and the value of travel time and the cross elasticity between the HSR demand and the conventional service fare. This specificity of the developed model results in a value of the option to defer that slightly diminishes with the increase of uncertainty. These findings can also be seen in Figure 8. It is always assumed that the discount rate remains unchanged as the volatility of the project changes.

Figure 8. The impact of both the volatility of the number of passengers and the discount rate
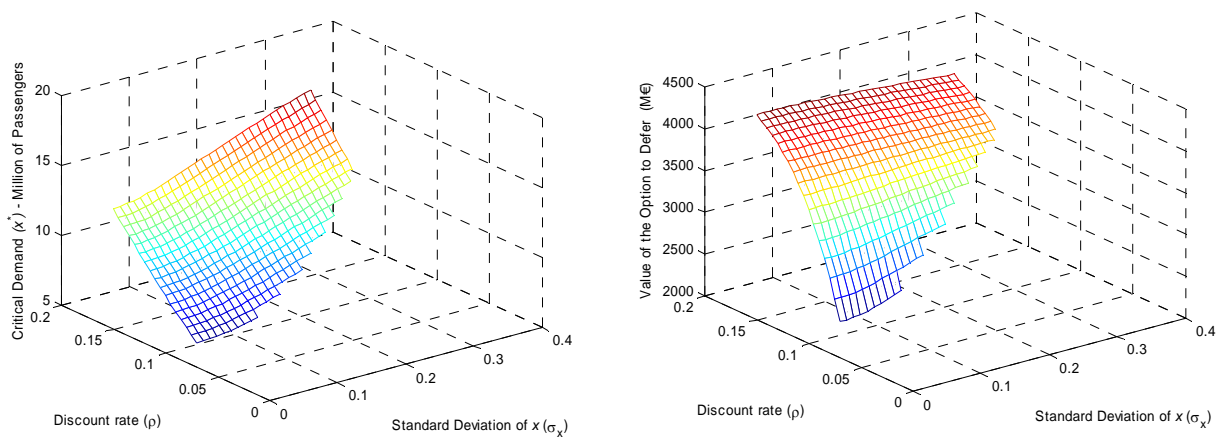

In case a larger period of time to implement the project is needed, the increase in uncertainty throughout time and the delay on the benefits from the investment's 
operation instigate a reduction in the investment's opportunity value and in the NPV (Figure 6).

Figure 9 gives the joint impact of both the discount rate $\rho$ and the investment expenditures $\gamma$ in the critical demand value $x^{*}$ and in the value of the option to defer. Both valuation outputs shows a direct relationship with these two parameters, turning the option to defer more valuable as this project parameters value increase. As showed in Figure 3 and Figure 4 this is due to a deeper decrease in NPV than the one registered in the investment's opportunity value.

Figure 9. The impact of both the investment expenditures and the discount rate
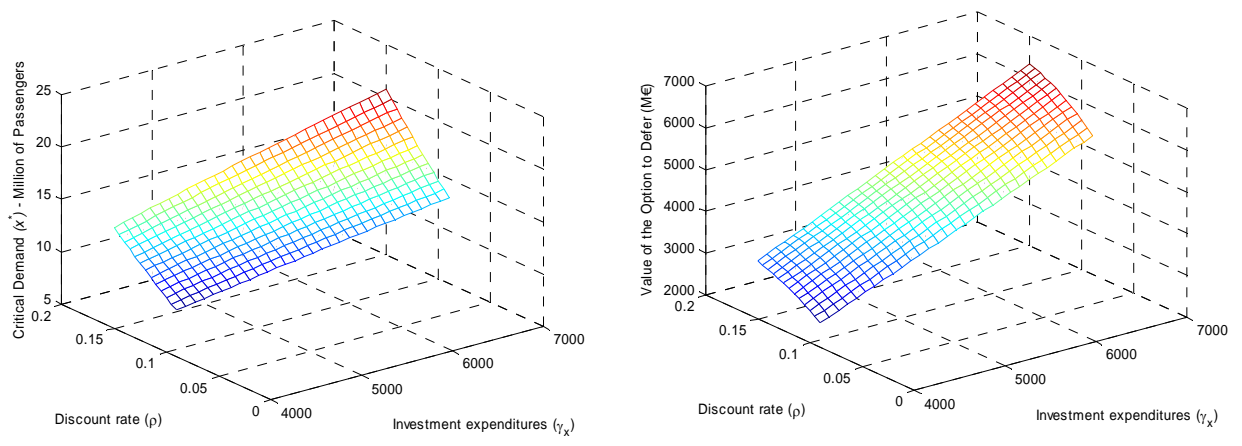

\section{Conclusion}

The present work develops a model aimed at finding the optimal timing to implement a HSR investment, in an uncertain environment. We have introduced several adjustments to the original valuation model of the option to defer (McDonald and Siegel, 1986) and to the optimal stopping model of Salahaldin and Granger (2005), given the need to design a model applicable to an HSR investment in an environment of stochastic demand. As far has we are aware, the development of closed form solution ROA's models to value railway investments was never done before.

The existence of a conventional railway service enables the analysis of the investment in HSR to be performed in an incremental basis, measured in terms of the corresponding utility functions. The indifference in the demand utility between HSR and conventional railway services makes possible for the problem to be equated in terms of finding a critical demand level that justifies the implementation of the investment. 
The presented developments, regarding the optimal timing to invest and the investment's opportunity value, have the advantage of offering a clear way to evaluate the utility of the HSR investment in each moment in time, for the set of potential users the society in general. The numerical example and simulation of some important input parameters demonstrates the consistency of the model concerning the behaviour of the valuation outputs.

In future research, it should be possible to enrich the model in order to include more uncertainty sources - like the fare price and the investment expenditure. Additionally, we expect to perform an empirical application ${ }^{1}$ capable of providing the feedback necessary to guide additional improvements in the structure of the modelling framework.

\section{References}

Bowe, M. and D. Lee (2004). Project Evaluation in the Presence of Multiple Embedded Real Options: Evidence From the Taiwan High-Speed Rail Project. Journal of Asian Economics, 15, 71-98.

Brandão, L. (2002). Uma Aplicação da Teoria das Opções Reais em Tempo Discreto para Avaliação de uma Concessão Rodoviária no Brasil. PhD Thesis, PUC-Rio.

Copeland, T. and V. Antikarov (2003). Real Options: A Practitioner's Guide. Thomson, Texere, New York.

Dixit, A. and R. Pindyck (1994). Investment Under Uncertainty. Princeton University Press, Princeton, New Jersey.

Majd, S. and R. Pindyck (1987). Time to Build, Option Value, and Investment Decisions. Journal of Financial Economics, 18 (1), 7-27.

Marathe, R e S. Ryan (2005). On the Validity of the Geometric Brownian Motion Assumption. The Engineering Economist, 50, 159-192.

McDonald, R. and D. Siegel (1986). The Value of Waiting to Invest. The Quarterly Journal of Economics, 101 (4), 707-728.

Myers, S. (1977). Determinants of Capital Borrowing. Journal of Financial Economics, $5(2), 147-175$.

\footnotetext{
${ }^{1}$ Portuguese public authorities have shown availability to release, to the authors, data regarding the new Portuguese rail link.
} 
Oksendal, B. (2003). Stochastic Differential Equations. An Introduction with Applications. Sixth Edition, Springer.

Owen, A. and G. Phillips (1987). The Characteristics of Railway Passengers Demand. Journal of Transport Economics and Policy, 21, 231-253.

Paxson, D. e H. Pinto (2005). Rivarly Under Price and Quantity Uncertainty. Review of Financial Economics, 14 (3-4), 209-224.

Pereira, P., A. Rodrigues e M. Armada (2006). The Optimal Timing for the Construction of an International Airport: a Real Options Approach with Multiple Stochastic Factors and Shocks. Real Options $10^{\text {th }}$ Annual International Conference, New York.

Rose, S. (1998). Valuation of Interacting Real Options in a TollRoad Infrastructure Project. The Quarterly Review of Economics and Finance, 38, 711-723.

Ross, S. (1996). Stochastic Processes. Second Edition, Wiley Series in Probability and mathematical Statistics, John Wiley \& Sons, Inc.

Salahaldin, L. e T. Granger (2005). Investing in Sustainable Transport to Relieve Air Pollution under Population-Growth Uncertainty. Real Options $9^{\text {th }}$ Annual International Conference, Paris.

Shilton, D. (1982). Modelling the Demand for High Speed Train Services. The Journal of Operational Research Society, 33, 713-722.

Smit, H. (2003). Infrastructure Investment as Real Options Game: The Case of European Airport Expansion. Financial Management, 32 (4), 27-57.

Trigeorgis, L. (1996). Real Options: Managerial Flexibility and Strategy in Resource Allocation. The MIT Press, Cambridge, MA.

Wardman, M. (1994). Forecasting the Impact of Service Quality Changes on the Demand for Inter-Urban Rail Travel. Journal of Transport Economics and Policy, 28, 287-306.

Wardman, M. (1997). Inter-Urban Rail Demand, Elasticities and Competition in Great Britain: Evidence from Direct Demand Models. Transport Research E, 33 (1), 15-18.

Wilson, G. (1986). Economic Analysis of Transportation: A Twenty-Five Year Survey. Transportation Journal, Fall, 33-44. 